Nawal A. Al-Sabawi BDS,MSc. (Lec.)

Sabah A. Ismail BDS,MSc. (Asst Prof.)

Raghad A. Al-Askary BDS,MSc. (Lec.)

\section{Effect of Different Techniques of Imme- diate and Delayed Post Space Preparation on Apical Seal}

\author{
Department of Conservative Dentistry \\ College of Dentistry, University of Mosul \\ Department of Conservative Dentistry \\ College of Dentistry, University of Mosul \\ Department of Conservative Dentistry \\ College of Dentistry, University of Mosul
}

$$
\begin{aligned}
& \text { الحخلاصة } \\
& \text { الأهداف: هدفت هذه الدراسة إلى دراسة تأثير التقنيات المستخلمة وأوقات إعداد الفضاء البلي على قابلية الحتم القمي لاثنان من أنواع حشوات الجذر المختلفة. }
\end{aligned}
$$

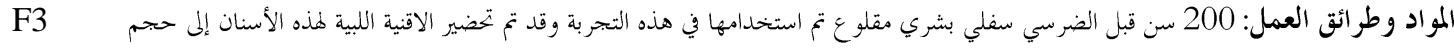

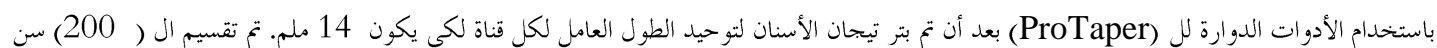

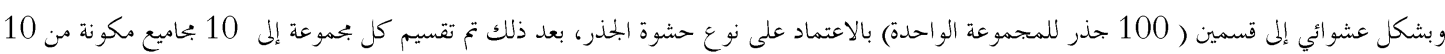

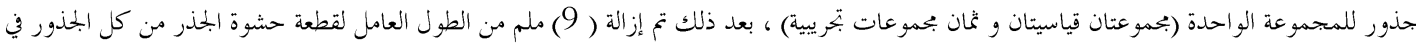

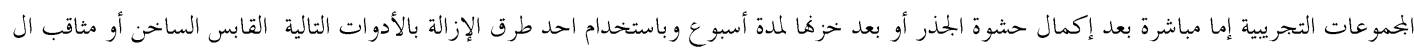

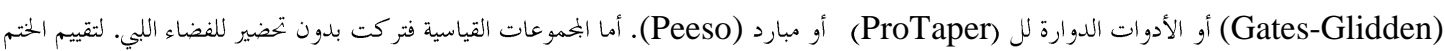

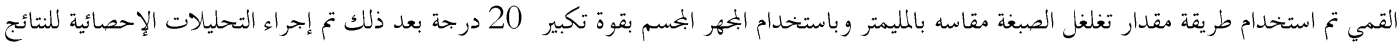

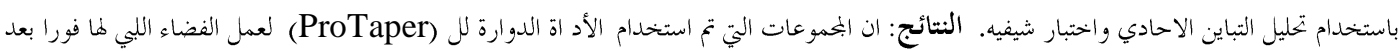

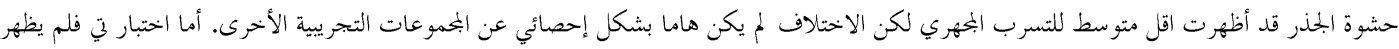

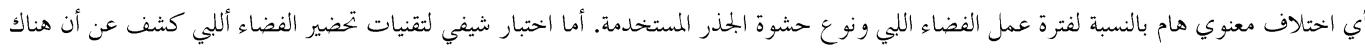

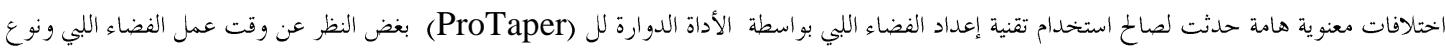

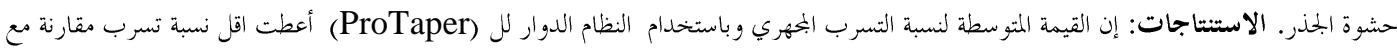

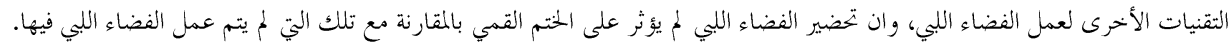

\title{
ABSTRACT
}

Aims: The purpose of this study was to investigate the effect of different post space preparation techniques and times on the apical seal of two types of gutta-percha obturation techniques. Materials and methods: Two hundred extracted human lower premolars were selected for this study. All teeth were decoronated to adjust the working length at $14 \mathrm{~mm}$. The canals were instrumented with ProTaper rotary instruments to size F3. The (200) root canals were randomly divided into two groups (100) of each according to the obturating method. Then each group was sub divided into (10) sub groups (10) of each ( 2 control groups and 8 experimental groups). Nine $\mathrm{mm}$ segment of gutta-percha was removed from the roots in each of the experimental groups either immediately after obturation or 7 days later using either heated pluggers, or Gates-Glidden drills or ProTaper rotary instruments or peeso reamers. The roots in the control groups were left without any post space preparation. The canal orifice of the roots in the experimental groups was sealed with amalgam. The extend of linear dye leakage was measured in $\mathrm{mm}$ with a stereomicroscope at (X 20). One-way ANOVA and sheffe statistical analysis were performed to the data collected. Results: The groups with ProTaper rotary instrument prepared the post space immediately after obturation( gps 3,11), showed the lowest mean leakage, but the difference was not statistically significant than the other experimental groups. T-test represented no significant differences existed at either two time interval studied and the type of obturation techniques. Sheffe-test for the post space preparation techniques revealed that there were a significant differences occurred with the use of ProTaper rotary instruments regardless the time interval and the obturation techniques. Conclusions: ProTaper rotary instrument provided less microleakage comparing to the other instruments used to prepare the post space. The post space preparation did not affect the apical seal in comparison with that of the intact filling.

Key words: apical microleakage, post space, post preparation. 
Al-Sabawi NA, Ismail SA, Al-Askary RA. Effect of Different Techniques of Immediate and Delayed Post Space Preparation on Apical Seal. Al-Rafidain Dent J. 2012; 12(2): 249-256.

Received: 2/1/2011 Sent to Referees: 4/1/2011

Accepted for Publication: 27/3/2011

\section{INTRODUCTION}

Endodontically treated teeth often lack sufficient support for a permanent restoration. An additional retention can be necessary gained from the root canal. Thus, these teeth often may require the use of an intra-canal post for retention of the core and to disperse the forces along the root. In order to create space for a post, part of the root canal filling material must be removed. ${ }^{(1,2,3)}$

Many techniques are used to remove gutta-percha, chemical using a solvent such as chloroform, thermal using hot endodontic pluggers, and mechanical, using a rotary instrument such as a Gates Glidden or a peeso reamer ${ }^{(2)}$. Recently, a nickel titanium (NiTi) drill with a non cutting tip and non cutting lateral surface has been proposed for removing gutta-percha and preparing the post space without enlarging the canal diameter like the other drills that might remove an excessive amount of root canal dentin and then reducing the strength of the root. ${ }^{(4)}$

During preparation of the post space the residual filling material may be dislodged, twisted or vibrated which creates a pathway for bacterial invasion and reinfection of the root canal system. Several factors can affect the integrity of the apical seal while post space is prepared such as length of remaining gutta-percha, time of removal of the filling material and method of gutta-percha removal. ${ }^{(1,5,6)}$

Few studies have investigated the effect of post space preparation on the integrity of the apical seal of the teeth obturated initially by different material and obturation technique..$^{(1,4)}$

There is no consensus on the time interval between the endodontic treatment and the post preparation with some authors proposing an immediate preparation and others recommending different time intervals. ${ }^{(7,8)}$

The procedures of preparing the post space are critical, and care is required to maintain the root canal seal and the aseptic conditions achieved with endodontics. ${ }^{(5,6,8)}$
The purpose of this study was to investigate the effect of different post space preparation techniques and times on the apical seal of two type of gutta-percha obturation techniques.

\section{MATERIALS AND METHODS}

Two hundred extracted human lower premolars of single root canal with mature apex were selected for this study, all teeth were scaled and polished and stored in distilled water at $37^{\circ} \mathrm{C}$ until use. The teeth were decoronated using diamond wheel saw (KG Sorensen SP, Brazil) under water coolant to make all canals $14 \mathrm{~mm}$ in length.

Then the roots were accessed, and the canal lengths were measured by inserting a size \# $15 \mathrm{k}$ - file (MANI, INC. JAPAN ) into each root canal until the tip of the file was visible at the apical foramen. The working length was established $1 \mathrm{~mm}$ short of the apex. All root canals were prepared at the predetermined working length with ProTaper (NiTi) rotary instrument to size F3. A total of 6 instruments were used with contra-angle rotary hand piece (Endo-Mate DT, INC., JAPAN). The speed of rotation was maintained at 250 rpm and torque $3 \mathrm{Nm}$. $2 \mathrm{ml}$ of $2.5 \%$ sodium hypochlorite $(\mathrm{NaOCl})$ solution was used for irrigation between each file size. ProTaper files were used according to the manufacturer's recommendations (each set of ProTaper files was used for 10 teeth). After the preparation procedure was complete, the teeth were irrigated with $3 \mathrm{ml}$ of $5.25 \%(\mathrm{NaOCl})$, and finally, the canals were dried with paper points ( Dentsply Maillefer, Switzerland), then randomly divided into two groups, each consisted of 100 samples according to the obturation methods (Matched-Taper Single Cone and Cold Lateral condensation of guttapercha). Tgadseal root canal sealer (Technical \& General LTD. London) was used as a sealer which then mixed and handled according to the manufacturers instructions and then the root canals were obturated using either size F3 single cone GP 
(Dentsply Maillefer, Switzerland) or by lateral condensation of size 30 master cone with accessory GP (Dia-Dent, Netherlands). Excess gutta-percha was then removed with a heated instrument and cold vertical compaction was performed with endodontic plugger.

Radiographs in buccolingual and mesiodistal directions were taken to confirm the quality of root canals obturations using a dental x-ray equipment (Trofy, France).

After that, each group was randomly subdivided into (4) sub groups, (two control groups of (10) roots in each and two experimental groups of (40) root in each). The root canals in the control groups left without post space preparation in which the microleakage was measured either immediately after obturation or 7 days after obturation. While the root canals in the experimental groups received post space preparation, either immediately after obturation or 1 week after root canal obturation to allow a complete setting of the sealer (for delayed groups the canal orifice was sealed with zinc phosphate cement (Drala Detax, GmbH \& co.KG) after obturation and storage in a distilled water in $100 \%$ relative humidity at $37^{\circ} \mathrm{C}$ ).

Then each of the experimental groups were further subdivided into 4 sub groups according to the method of post space preparation (10 of each). The final groups were (20) group of 10 samples in each (16 experimental and 4 control) groups.

Nine $\mathrm{mm}$ segment of gutta-percha would be removed from each root canal obturation in the experimental groups using the following instruments:

- Heated-endodontic pluggers (JoHnQuayle, England) size 30, 25, 20 (Figure 1) respectively used to removed the guttapercha incrementally until $5 \mathrm{~mm}$ of apical gutta-percha was remained.

- Gates-Glidden drills (Dentsply Maillefer, Switzerland) size 1, 2 and 3 (Figure 2) coupled to a slow-speed hand-piece (W\&H, Strauts, Austria) which was introduced into the canal to the predetermined length, then the drill was inserted and withdrawn several times with rotation movement until the filling material had been completely removed from the canal walls, and leaving $5 \mathrm{~mm}$ of remaining fill- ing (each drill was used 5 times).

- ProTaper (NiTi) rotary instrument SX file (Figure 3) are used to remove the gut ta-perch as describe with Gates-Glidden drills.

- Peeso reamers ( Dentsply, swiss made, Ballaigres) size 1-3 (Figure 4) coupled with slow speed hand piece to remove the gutta-percha as describe with GatesGlidden drills.

Then a rubber stopper was placed on the spreader to verify depth of the instrument within the canal and ensure that 5 $\mathrm{mm}$ of intact GP remained in the apical portion of the root canal. The remaining apical GP was gently vertically condensed with the endodontic plugger of appropriate size, then a radiographs were taken to ensure the adequacy of the apical seal after post space preparation. When the preparations were completed, each root canal irrigated with $3 \mathrm{ml}$ of $5.25 \%(\mathrm{NaOCl})$, dried, then a small cotton pellet was placed 6 $\mathrm{mm}$ deep in to the canal to act as a base for the coronal material, then the orifice of the root access opening was closed with $3 \mathrm{~mm}$ depth of amalgam.

The surfaces and coronal opening of all root canals were then covered with sticky wax and coated with two layers of nail varnish except for the apical $2 \mathrm{~mm}$. The experimental and control groups were each placed in numbered containers which were filled to identical levels with $2 \%$ methylene blue dye for 7 days. Then the teeth were rinsed in running water to remove excess dye and dried.

The roots were grooved buccally and lingually with a diamond wheel saw (KG Sorensen SP, Brazil) under water coolant, ensuring that the root canal filling was not penetrated, and then they were split into two halves by levering with a plaster knife. The linear extent of dye penetration from the apical root ends to the deepest extent of dye penetration into the coronal portion was measured in mm using a stereomicroscope (Motic, TAIWAN) at (X 20 magnification) by two observers and the average of the two reading was recorded. The data was statistically analyzed to determine if there were statistically significant differences among groups. 

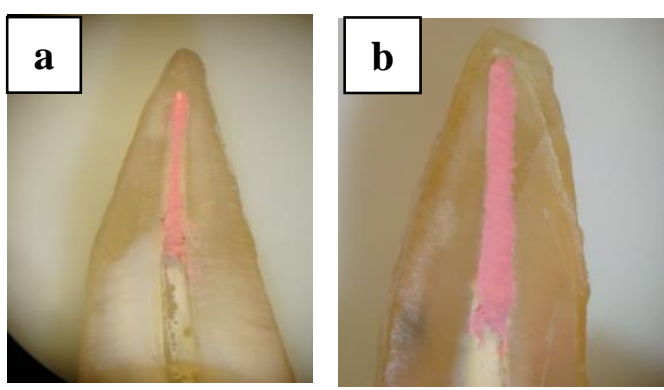

Figure (1): post space preparation with hot endodontic pluggers (a) Cold Lateral condensation gutta-percha (b) Match SingleCone gutta-percha.
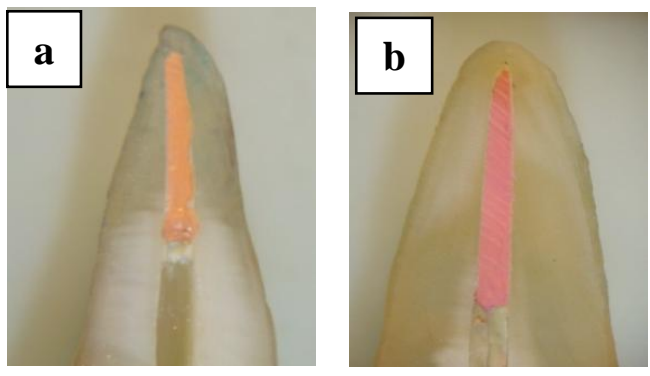

Figure (3): post space preparation with ProTaper (NiTi) rotary system (a) Cold Lateral condensation gutta-percha (b) Match Single-Cone gutta-percha.

\section{RESULTS}

Table (1) showed the mean apical leakage and standard deviations of the experimental and control groups. One-way analysis of variance (Table -2) was performed among the groups and revealed that there was no significant differences at $(p=0.05)$ between the control and the experimental groups. Although, group (11: 1.i.pr) had the least amount of mean microleakage $(0.310 \pm 0.338)$, but the difference was not statistically significant, while group (5: s.d.h) show the highest microleakage mean $(0.820 \pm 0.637)$.

T-test for the type of root canal obturation and for the time of post space preparation (Table $-3,4)$ respectively represent
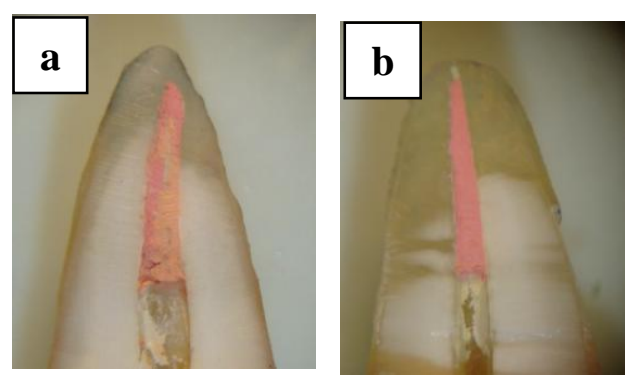

Figure (2): post space preparation with gates-glidden drills (a) Cold Lateral condensation gutta-percha (b) Match SingleCone gutta-percha.
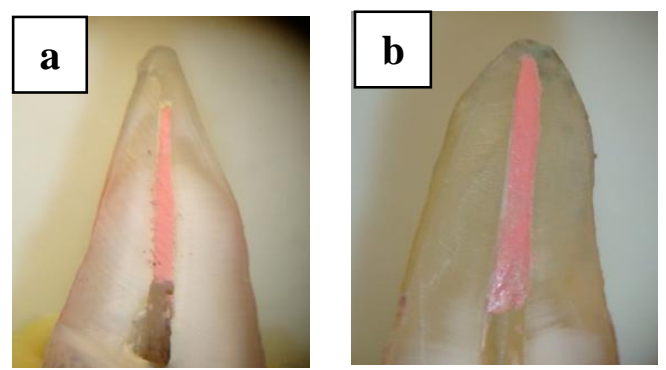

Figure (4): post space preparation with peeso reamers (a) Cold Lateral condensation gutta-percha (b) Match Single-Cone gutta-percha.

no statistically significant differences among the groups at $(\mathrm{p}=0.05)$.Table (5) showed the mean apical leakage and standard deviations for the effect of post space preparation techniques on the apical leakage.

One-way ANOVA for the effect of post space preparation techniques (Table 6) represented a significant difference in apical leakage among the experimental groups. Sheffe-test for comparing the four method of post space preparation (Table 7) revealed that the least leakage was detected for groups with ProTaper (NiTi) rotary system regarding the type of obturation technique and the time interval for the post space preparation. 
Table (1): The mean and standard deviations in $\mathrm{mm}$ of the experimental and control groups.

\begin{tabular}{llllc}
\hline \multicolumn{2}{c}{ Groups } & N & Mean & Std. Deviation \\
\hline Gp.1 & (s.i.h) & 10 & 0.640 & 0.476 \\
Gp.2 & (s.i.g) & 10 & 0.540 & 0.419 \\
Gp.3 & (s.i.pr) & 10 & 0.370 & 0.334 \\
Gp.4 & (s.i.pe) & 10 & 0.630 & 0.459 \\
Gp.5 & (s.d.h) & 10 & 0.820 & 0.637 \\
Gp.6 & (s.d.g) & 10 & 0.630 & 0.507 \\
Gp.7 & (s.d.pr) & 10 & 0.640 & 0.452 \\
Gp.8 & (s.d.pe) & 10 & 0.650 & 0.417 \\
Gp.9 & (l.i.h) & 10 & 0.770 & 0.569 \\
Gp.10 & (l.i.g) & 10 & 0.560 & 0.406 \\
Gp.11 & (l.i.pr) & 10 & 0.310 & 0.338 \\
Gp.12 & (l.i.pe) & 10 & 0.600 & 0.439 \\
Gp.13 & (l.d.h) & 10 & 0.710 & 0.566 \\
Gp.14 & (l.d.g) & 10 & 0.640 & 0.503 \\
Gp.15 & (l.d.pr) & 10 & 0.440 & 0.485 \\
Gp.16 & (l.d.pe) & 10 & 0.660 & 0.392 \\
Gp.17 & (c.s.i) & 10 & 0.330 & 0.286 \\
Gp.18 & (c.s.d) & 10 & 0.390 & 0.276 \\
Gp.19 & (c.l.i) & 10 & 0.320 & 0.278 \\
Gp.20 & (c.l.d) & 10 & 0.330 & 0.290 \\
Total & & 200 & 0.549 & 0.448 \\
\hline cone GP & l= lateral condensation GP & \multicolumn{2}{c}{ i= immediate } \\
endodontic plugger & g= gates-glidden drill & pr= ProTaper (NiTi) rotary instrument
\end{tabular}

$\mathrm{h}=$ hot endodontic plugger $\mathrm{g}=$ gates-glidden drill $\mathrm{pe}=$ peeso reamers $\quad \mathrm{c}=$ control

Table (2): One-way analysis of variance of the experimental and control groups.

\begin{tabular}{cccccc}
\hline & Sum of squares & df & Mean Square & Fc & Ft \\
\hline Between Groups & 4.862 & 19 & 0.256 & 1.312 & 10571 \\
Within Groups & 35.098 & 180 & 0.195 & & \\
Total & 39.960 & 199 & & & \\
\hline
\end{tabular}

$\mathrm{Fc}=\mathrm{F}$ calculated

$\mathrm{Ft}=\mathrm{F}$ tabulated

Table (3): T-test for the effect of type of obturation method on the apical seal.

\begin{tabular}{cccccc}
\hline Groups & $\mathbf{N}$ & Mean & Std.Deviation & Tc & Tt \\
\hline S & 100 & 0.564 & 0.448 & 0.472 & 1.96 \\
L & 100 & 0.534 & 0.449 & & \\
\hline S= Single Cone GP & L= Lateral Condensation GP & Tc= T calculated & & Tt= T tabu-
\end{tabular}

lated

Table (4): T-test for the effect of two time interval.

\begin{tabular}{cccccc}
\hline Groups & $\mathbf{N}$ & Mean & Std.Deviation & Tc & Tt \\
\hline I & 100 & 0.507 & 0.420 & 1.328 & 1.96 \\
D & 100 & 0.591 & 0.472 & & \\
\hline I= Immediate & $\mathrm{D}=$ Delayed & \multicolumn{2}{c}{$\mathrm{Tc}=$ T calculated } & $\mathrm{Tt}=\mathrm{T}$ tabulated &
\end{tabular}


Table (5): The difference in mean and standard deviation for the effect of post space prepara tion techniques on the apical leakage.

\begin{tabular}{cccc}
\hline Groups & $\mathbf{N}$ & Mean & Std.Deviation \\
\hline Hot pluggers & 40 & 0.735 & 0.548 \\
Gates-gildden drills & 40 & 0.592 & 0.446 \\
ProTaper rotary in- & 40 & 0.440 & 0.412 \\
struments & & & \\
Peeso reamers & 40 & 0.635 & 0.425 \\
Total & 160 & 0.600 & 0.469 \\
\hline
\end{tabular}

Table (6): One- way analysis of variance for the effect of post space preparation method on apical leakage.

\begin{tabular}{cccccc}
\hline & Sum of squares & Df & Mean square & Fc & Ft \\
\hline Between groups & 1.804 & 3 & 0.601 & 2.834 & 2.600 \\
Within Groups & 33.106 & 156 & 0.212 & & \\
Total & 34.910 & 159 & & & \\
\hline
\end{tabular}

$\mathrm{Fc}=\mathrm{F}$ calculated $\quad \mathrm{Ft}=\mathrm{F}$ tabulated

Table (7): Sheffe-test for comparing the method of post space preparation.

\begin{tabular}{ccccc}
\hline & Hot plugger & $\begin{array}{c}\text { Gates-gildden } \\
\text { drill }\end{array}$ & $\begin{array}{c}\text { ProTaper rotary } \\
\text { instrument }\end{array}$ & Peeso remear \\
\hline Hot pluggers & - & 1.915 & $8.201^{*}$ & 0.943 \\
Gates-gildden drills & - & - & 2.194 & 0.170 \\
ProTaper rotary in- & - & - & - & 3.587 \\
struments & - & - & - & - \\
Peeso reamers & - & - & & \\
\hline
\end{tabular}

Sh. Tabulated $=7.8$ on $\mathrm{p}=0.05 \quad *=$ significant for ProTaper rotary instrument

\section{DISCUSSION}

The restoration of root treated teeth often requires the use of posts, their use may affect the quality of the seal of the root canal filling. Post space preparation has been reported to reduce the sealing capacity of apical endodontic obturating material, since during the preparation of this space, the root filling material may be dislodged creating voids in the obturation, and increasing the possibility of leakage. ${ }^{(1,7)}$

In the present study, post space preparation did not affect the apical seal quality, which did not different statistically from that in groups without this space preparation.

Several studies have reported that no statistical difference in apical leakage could be observed before or after post preparation with various techniques. ${ }^{(6,9)}$ In the study reported here, there was no significant dif- ference in the sealing ability of the remaining root filling material related to the obturation techniques or the timing of post space preparation.

$\mathrm{Li}$ et $a l^{(9)}$ represented that the group with single cone obturation technique had the lowest microleakage than the group with lateral compaction technique in postdowel space preparation.

Aydemir et al ${ }^{(1)}$ and Pesce et al ${ }^{(7)}$ showed that delayed removal of GP for post space preparation produced numerically better results in apical leakage, but without statistical significance compared to immediate preparation since before complete sealer setting is achieved, it is possible that the filling become twisted or vibrate during mechanical post space preparation, in such a way to cause disruption of the apical seal. 
Solano et al ${ }^{(10)}$ showed significantly less leakage when the post space was prepared at the time of obturation than when it was prepared at one week after obturation, since the sealer is still within its working time when the gutta-percha is removed and defects can be remedied. When post space preparation is delayed, the sealer sets completely, such that removal of the filling mass later on causes movement that disrupts the bond sealer interface. ${ }^{(8,10)}$

In the present in vitro study, the effect of different post space preparation techniques on the apical seal was evaluated and the results revealed that the least leakage was detected for groups with ProTaper (NiTi) rotary system regarding the type of obturation technique and the time interval for the post space preparation.

Salke et $a l^{(3)}$ represented no statistically significant difference in apical dye leakage between flame - heated endodontic pluggers and peeso reamers when $5 \mathrm{~mm}$ of apical gutta-percha seal remained, and NiTi rotary instrument group showed lowest mean leakage but the difference was not statistically significant.

Flame-heated endodontic pluggers are a fast and inexpensive means of guttapercha removal and there is no canal shape alteration but the burning potential for the dentist, dental assistant or the patient may happen during its use, also length control can be a problem because the rubber stopper for canal depth measurement may catch fire or degrade so rapidly that it slides freely on the plugger. ${ }^{(2,3)}$

Peeso reamers and gates-glidden drills are inexpensive and remove the guttapercha very rapidly, the rotary action creates parallel walls which provide optimal retention for the post. But root perforation, canal transportation, over enlargement of the canal resulting in weakened remaining root structure, and instrument separation may occur during post space preparation. ${ }^{(11)}$

Balto et $a l^{(2)}$ revealed that the removal of gutta-percha with peeso- reamer resulted in less disturbance of the apical seal than the removal with hot plugger.

The specific flute design and rotary motion of the ProTaper tend to pull guttapercha in to the file flutes and direct it towards the canal orifice. Although this sys- tem was fast and effective in post space preparation but instrument separation could be happen if the instrument bound in a canal irregularity or was used past a canal curvature. ${ }^{(12)}$

With regard to the cast-dowel preparation technique, various studies demonstrated in vitro that the seal of the remaining obturating material was not compromised when rotary instruments were used because the frictional heat produced by rotary instrument plasticized the gutta-percha and removed it without excessive pull in addition slight apical pressure during the removal of gutta-percha may act as vertical condensation which might improve the apical seal. ${ }^{(2,4)}$ In contrast, other authors claimed that the apical seal was worse after preparation with rotary instruments comparing to the seal of completely obturated root canals. ${ }^{(6)}$

Grecca et al ${ }^{(8)}$ represented that there was no significant difference in the sealing ability of the remaining root filling material related to the technique or the timing of post space preparation.

Hebeishi et al ${ }^{(13)}$ represented significantly higher microleakage when guttapercha was removed by gates-glidden drills at 7 days as compared to immediate removal and there was no significant difference in microleakage between hot pluggers and gates-glidden burs at either time periods.

\section{CONCLUSIONS}

Within the parameters of this in vitro leakage study, the following conclusions can be drawn. Post space preparation do not affect the seal ability of root canal obturation. The apical seal of the remaining root canal filling was not affected by the type of obturation technique and time of post space preparation, but rather with the methods of post space preparation.

The use of ProTaper (NiTi) rotary instruments for post space preparation provide significantly better apical seal compared to the hot pluggers, gates-glidden drills, and peeso reamers.

\section{REFERENCES}

1. Aydemir H, Ceylan G, Tasdemir T, Kalyoncuoglu $\mathrm{E}$ and Isildak I. Effect of immediate and delayed post space prep- 
aration on the apical seal of root canals obturated with different sealers and techniques. J Appl Oral Sci. 2009; 17: 605-610.

2. Balto H, Al- Nazhan S, Al-Mansour K, Al- Otaibi M and Siddiqu Y. Microbial leakage of Cavit, IRM, and Temp Bond in post-prepared root canals using two methods of gutta-percha removal: An invitro study. $J$ Contem Dent Pract. 2005; 6: 1-6.

3. Salke S, Shivamurthy G and Shivanna V. Permeability of remaining endodontically obturated material after postspace preparation using different techniques of gutta-percha removal- An in vitro study. J Endod. 2009; 21:36-40.

4. Coniglio I, Magni E, Goracci C, Radovic I, Carvalbo C, Grandini S and Ferrari M. Post space cleaning using a new nickel titanium endodontic drill combined with different cleaning regimens. JOE. 2008; 34: 83-86.

5. Mosbonov J, Goldberg I, Gottlieb A and Peretz B. The effect of distance between post and residual gutta-percha on the clinical outcome of endodontic treatment. JOE. 2005; 31: 177- 197.

6. Abramovitz I, Lev R, Fuss Z and Metzger $Z$. The unpredictability of seal after post space preparation: a fluid transport study. J Endod. 2001; 27:292-295.

7. Pesce A, López S and Rodriguez M. Effect of post space preparation on apical seal Influence of time interval and sealer. Med Oral Patol Oral Cir Bucal. 2007; 12: 464-468.

8. Grecca F, Rosa Â, Gomes M, Parolo C, Bemfica $\mathbf{J}$ and Frasca L. Effect of timing and method of post space preparation on sealing ability of remaining root filling material: In vitro microbiological study. JADA. 2009; 75: 583-583e.

9. Li Jh, Ma Cb, Zhang X, Zhu Gq, Chen Jp and Tang Xc. Effect of post space preparation on the seal ability of root canal filled with 3 filling methods. Shanghai Kou Qiang Yi Xue. 2009; 18: 639-642.

10. Solano F, Hartwell G and Appelstein C. Comparison of apical leakage between immediate versus delayed post space preparation using AH Plus sealer. $J$ Endod. 2005; 31: 752-754.

11. Kuttler S, McLean A, Dorn $S$ and Fischzang A. The impact of post space preparation with gates-glidden drills on residual dentin thickness in distal roots of mandibular molars. J Am Dent Assoc. 2004; 135: 903-909.

12. Gu Ls, Ling JQ, Wei X and Huang XY. Efficacy of protaper universal rotary retreatment system for gutta-percha removal from root canals. Int Endod $J$. 2008; 41: 288-295.

13. Hebeishi A, Tawfik $\mathrm{H}$ and Hashem A. Longitudinal evaluation for the effect of post space preparation on microleakage of obturation root canal. Egypt Dent Associ. 2007; 53: 1193(abstract). 